\title{
Co-Designing for Privacy, Transparency, and Trust in K-12 Learning Analytics
}

\author{
June Ahn \\ junea@uci.edu \\ University of California - Irvine \\ Irvine, CA, USA
}

\author{
Fabio Campos \\ fabioc@nyu.edu \\ New York University \\ New York, NY, USA
}

\author{
Ha Nguyen \\ thicn@uci.edu \\ University of California - Irvine \\ Irvine, CA, USA
}

\author{
Maria Hays \\ mehays@uw.edu \\ University of Washington \\ Seattle, WA, USA
}

\author{
Jan Morrison \\ jan.morrison@vanderbilt.edu \\ Vanderbilt University \\ Nashville, TN, USA
}

\begin{abstract}
The process of using Learning Analytics (LA) to improve teaching works from the assumption that data should be readily shared between stakeholders in an educational organization. However, the design of LA tools often does not account for considerations such as data privacy, transparency and trust among stakeholders. Research in human-centered design of LA does attend to these questions, specifically with a focus on including direct input from K-12 educators. In this paper, we present a series of design studies to articulate and refine conjectures about how privacy and transparency might influence better trust-building and data sharing within four school districts in the United States. By presenting the development of four sequential prototypes, our findings illuminate the tensions between designing for existing norms versus potentially challenging these norms by promoting meaningful discussions around the use of data. We conclude with a discussion about practical and methodological implications of our work to the LA community.
\end{abstract}

\section{CCS CONCEPTS}

\section{- Human-centered computing $\rightarrow$ Participatory design.}

\section{KEYWORDS}

Dashboards, K-12 Education, Ethics, Privacy, Co-Design, HCI

\section{ACM Reference Format:}

June Ahn, Fabio Campos, Ha Nguyen, Maria Hays, and Jan Morrison. 2021. Co-Designing for Privacy, Transparency, and Trust in K-12 Learning Analytics. In LAK21: 11th International Learning Analytics and Knowledge Conference (LAK21), April 12-16, 2021, Irvine, CA, USA. ACM, New York, NY, USA, 11 pages. https://doi.org/10.1145/3448139.3448145

\section{INTRODUCTION}

In early 2019, a group of colleagues from our research group warned our team about the risk of losing all users of our experimental dashboard, in one of our partner districts, if data privacy concerns were

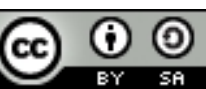

This work is licensed under a Creative Commons Attribution-ShareAlike International 4.0 License.

LAK21, April 12-16, 2021, Irvine, CA, USA

(C) 2021 Copyright held by the owner/author(s)

ACM ISBN 978-1-4503-8935-8/21/04.

https://doi.org/10.1145/3448139.3448145 not addressed. This specific problem of practice spurred an intensive co-design process of a data privacy module capable of not only keeping our partners engaged but promoting greater transparency, trust and knowledge about data use in the $\mathrm{K}-12$ school context. This paper provides a thick design narrative of this process and offers a discussion of its implications for ethical LA design.

Our study happens within a larger network of three researchpractice partnerships (RPPs) between universities and K-12 school districts in the United States. Within the RPPs, our team co-designed a data dashboard with partner Mathematics educators that displays survey data collected from students, describing their experiences with in-class collaboration, discussion and Mathematical thinking. In designing the privacy module of said dashboard, our team collaborated with teachers, instructional coaches and district leaders.

K-12 schools and districts often have regulatory obligations to protect students' privacy and safety when using digital technologies $[1,17]$. In this context, issues of ethics and privacy are topics of increasing focus for Learning Analytics (LA) scholars and designers $[11,12,34-36,38]$. While the LA community has developed principles and codes of ethical practice, little is known about how to actually reify such principles through human-centered design work [4], especially with the direct input of stakeholders [28]. A key principle found in LA codes of practice is to communicate transparently with stakeholders about issues of privacy, data collection and data use. What such codes cannot explain, however, is how providing information to the user might lead to perceived transparency and trust, and whether this potential perception may actually encourage users to share their data within an education context. This gap in our field's literature motivated our choice for iterative design work with educators.

In addition, emerging LA scholarship has focused on designing tools that attend to ethical concerns, but without specifically addressing K-12 schools as main sites of practice [5]. We contribute to this body of work by documenting a series of participatory design iterations, where we engaged with $\mathrm{K}-12$ educators to examine how privacy, transparency, and trust dynamics around data sharing and analytics played out in specific school settings, and how we could re-imagine tools to address these dynamics. With this objective in mind, our team set forth to explore the following question: What might an iterative design process reveal about privacy, transparency and trust in K-12 schools? 
This paper makes two contributions. First, understanding that design-based studies are still relatively new to the LA community [30], we provide a detailed account of how the development of a tool - a data privacy and sharing workflow within a LA dashboard - reveals and challenges norms around data privacy, transparency, and trust in K-12 settings. We employ methodologies typically found in the Human-Computer Interaction (HCI) and the Learning Sciences (LS) fields and present our findings through thick design narratives [14]. Second, we make a methodological contribution to the emerging topic of human-centered LA [4]. Using a a designas-research framework [45] and conjecture maps - a conceptual tool that traces design trajectories based on a set of assumptions $[31,40]$ - we discuss how material aspects of prototypes (e.g. the tool, associated tasks, etc.) are theoretically linked to key mediating processes among educators (e.g. facilitating conversations about data), and how such processes might lead to more transparent data sharing in the future. For this reason, we refrain from adopting an evaluative approach and focus on what such a design process might contribute to our field and reveal about K-12 schools.

\section{THEORETICAL BACKGROUND}

\subsection{Privacy, Transparency and Trust}

In the United States, using data to inform instruction has become a standard expectation for K-12 educators. However, data use in schools is a complex process, likely shaped by social and political dynamics in school organizations [10,32]. Similarly, little attention has been paid to understanding how to mitigate the potential lack of trust and privacy that are present in K-12 organizations, that may impact data use in schools. Using data to inform future instruction is hardly an individual endeavor. Numerous collective data-use practices take place at schools, such as inquiry cycles [20,21] organized cycles of inquiry where educators analyze data relating to an agreed upon problem of practice - and instructional coaching $[3,23]$, where experienced educators "coach" other teachers towards school or district-level goals.

Data use in schools, as a fundamentally social process, influences relationships and dynamics among educators (e.g. teachers, instructional coaches, principals etc.) [19]. Key factors such as information ownership, hierarchy, and purpose of data use might make or break systems for data-driven decision making in a K-12 school. A culture of compliance - where data is collected at the school but used at the district or state level - might damage trust between teachers and other stakeholders, hamper schools' ability to change instructional delivery [10] and even shape the focus teachers have when approaching learning data [15].

Extant research shows that information about privacy and security is not widely available in a school, and educators often have to rely on peers to vet decisions related to their privacy concerns [17]. In fact, teachers' collegial, interpersonal relationships have been described as a key influence on decisions surrounding how data is used and shared among school educators [18]. Hence, we saw a need to design not only tools but also practices and discourses [31] that incorporate, align or even challenge those relationships. As proposed by Kumar and colleagues [17], making issues of privacy and security more visible within K-12 education technologies should be a goal of LA and HCI designers.

\subsection{Designing for Privacy and Transparency}

Scholars have long understood that any designed tool necessarily carries the choices and intentionalities - or "politics" - of its creators [41]. Several studies have tried to establish frameworks and paradigms for incorporating privacy protections in digital tools $[39,42,43]$. One example is Privacy by Design (PBD), a model that adopts a proactive, rather than reactive, stance for integrating privacy into information-rich tools. For example, Wong and colleagues [43] use a PBD approach to directly engage target users of a tool to "identify potential issues related to privacy and discuss ways to address those conflicts" (p.3). The authors suggest that design may act as a value lever to promote discussions around privacy and trust among users. Their work also illuminates how privacy values, concerns and expectations are related to users' positioning in a particular context or social setting, a notion that is echoed by our own findings below.

A similar concern with ethical design can be found in the work of Verhagen and colleagues [39], who employed the Value Sensitive Design (VSD) framework to design educational tools. VSD is a set of design norms and philosophies concerned with how to represent users' values, needs and concerns in a particular tool, as well as concerned with the potential impacts and implications of such tools over their main stakeholders. Verhagen and his team found that attuning to users' values helped designers navigate the trade-offs between transparent privacy policies, learning goals, technical requirements and the usability of the tool. A key way to deal with privacy trade-offs is by employing participatory and critical design practices. Wong and colleagues [42] suggested that employing such practices would allow designers to go beyond compliance and checklists - which typically reify legal requirements - to encourage deeper and more holistic reflections based on users' values and representations of ethics and privacy.

Within the LA community, Chen and Zhu's [5] application of VSD and other design methods of research illuminated how designed features may support or hinder certain stakeholder values and practices. In one of their studies, the authors developed design conjectures based on tensions between student privacy and a tool's usability and goal. Similarly, Prestigiacomo et al.s [28] work encouraged teachers to convey their own ideas about what type of information is relevant, and ensuring their accountability for data-driven decisions. In our study, we build on this nascent body of work by developing conjectures about how privacy, transparency, and trust can be enacted with K-12 educators, that may be tested and refined through participatory design.

\subsection{Privacy and Ethics in LA scholarship}

The LA community has explored issues of ethics since its inception $[9,11-13,35]$. One emerging observation is that professional relations among school employees, such as teachers, coaches and district leadership, might be affected by the widespread collection and use of learning data. When LA practice is entwined with school governance, analytics might be converted into performance indicators and thus serve evaluative - and not learning - purposes. As Griffiths [13] posits, "whether or not learners and teachers are keen to use an LA intervention, they may nevertheless find that they have to do so if they are to maintain their studies or jobs" 
(p. 50). Similarly, Slade and Prinsloo [35] discussed how the collection and use of data might negatively influence relations among LA stakeholders, and urged the community to promote reciprocal transparency between learners, instructors and school leadership.

Another common finding is that understandings of ethical issues are transient and highly situated in the context of use. Ferguson and colleagues [11] argue that the beliefs of LA users, designers and researchers must always be considered, especially when they relate to "power, surveillance, and the purpose of school" (p. 7) Likewise, Slade, Prinsloo and Khalil [36] suggest that trust in the data system, and the perceived benefits of sharing one's data, are not static but in constant negotiation. These conclusions stress the importance of designing LA tools not just for but with the direct input of stakeholders.

To open space for LA tools that are responsive to different contexts, LA researchers established several frameworks, and codes of ethical practice [9, 27, 33, 35]. Drachsler and Greller's [9] DELICATE model, for example emphasizes the need for transparency in LA tools: stakeholders need to be involved and properly informed about issues of data collection and use. Similarly, Sclater [33] described the creation of a code of practice for LA which allowed for all stakeholders, including those whose data was being collected, to understand why and how information was being used.

However, such frameworks fall short in explaining how providing information can lead to perceived transparency, as well as how - or whether - transparency might lead to better data sharing practices in a school. Additionally, LA research has still not illuminated which mechanisms - such as tasks and practices enabled by a particular tool - might be conducive to increased trust - or the willingness to rely in a partner [26] among peers in a data system. This gap motivated our choice for iterative participatory design work.

\section{METHODS}

\subsection{Context of Investigation}

This study is situated within a larger research-practice partnership (RPP) called PMR2 - Practical Measures, Routines and Representations - that involves three school districts (hereby refered to as North, South and West), and four universities in the United States. Our network of partners work together to: (1) develop practical measures $[16,44]$, which capture process data such as student thinking in middle school Math classrooms, and (2) support teachers to improve their facilitation of student-centered discussion of math concepts using this data. Practical measures are fast and frequent indicators of how students experience instruction, carefully crafted to provide contextualized information and support instructional improvement [16]. Teachers collect practical measure data through student-facing surveys, administered in various moments of instruction. For example, students might evaluate their experience in participating in a small group discussion activity by taking an in-class survey, and answering questions such as "who talked the most during today's discussion?" [16].

In our partner districts, coaches support teachers by promoting data-driven inquiry about concrete aspects of instruction. This process often utilizes graphical representations of practical measure data collected from students. Such data is also typically shared with district-level math specialists and master-coaches to inform broader,

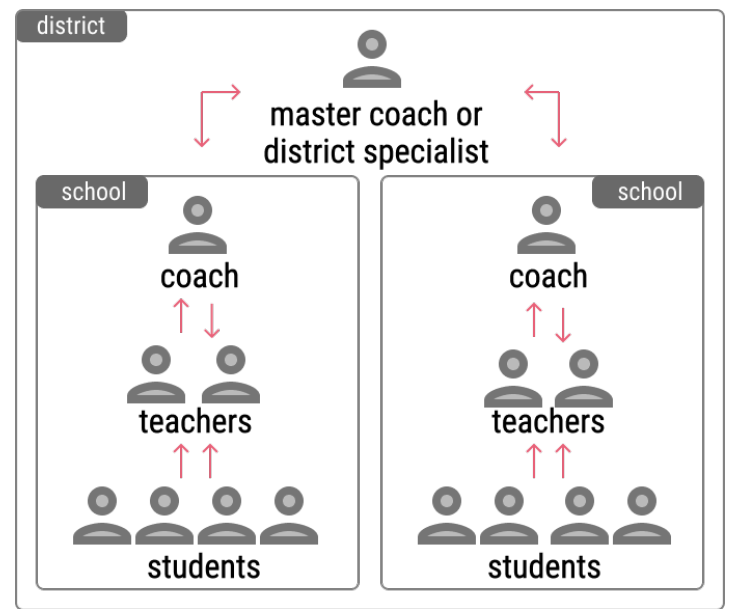

Figure 1: Data flow within schools and districts.

district-wide initiatives (Figure 1). As part of PMR2, our design team continually co-designs a dashboard application to support our partner educators' use of practical measure data. Figure 2) shows one of the interactive graphs of student data produced by our tool. Here we note that nuanced decisions about the interface elements, come directly from a research-practice partnership plus co-design process with partners, and these seemingly minute decisions align with in-depth analyses of our partners' workflows, organizational cultures, and analysis needs [2].

\section{Q.6 | What was the purpose of today's whole class discussion?}

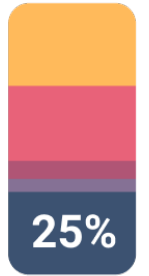

$1 / 28 / 19$

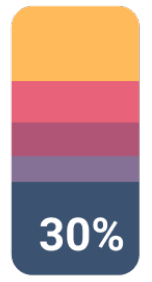

$2 / 11 / 19$

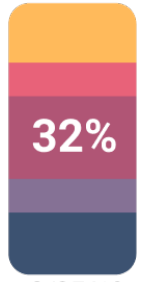

$2 / 25 / 19$

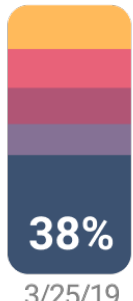

$3 / 25 / 19$
Share how we solved problems using steps our teacher taught us

Learn the way the teacher showed us to solve the problem

Learn ways to solve a problem from other students

Share a mathematical idea we came up with on our own

Check to see if answers were correct

Figure 2: A sample graph of our dashboard, displaying student survey data about in-class Math discussions.

Since the first meetings of the PMR2 network, researchers and district leaders voiced that teachers would refrain from using the 
Table 1: Data sources utilized in the study

\begin{tabular}{lll}
\hline Event & Date & Data Corpus \\
\hline Co-design sessions 1, 2 and 3 & May, 2020 & 3 transcripts, 3 1-hr videos, 12 pages of notes. \\
RPP Progress Report Meeting & March, 2020 & 1 transcript, 1hr video, 6 pages of notes. \\
Weekly meeting notes & April-June, 2020 & 2 pages of notes + 7 slides \\
Design Notes (Figma) & April-July 2020 & Approximately 40 prototype notes \\
RPP Annual Meetings & July 2018, 2019,2020 & 12 pages of notes, 6 posters, 2 1-hr videos \\
Site visit (North District) & September 2019 & 1 transcript, 1hr video, 3 pages of notes. \\
\hline
\end{tabular}

dashboard if they thought it could be used for evaluative purposes. In early 2019, researchers embedded in district "West" warned our team about the risk of losing all users of that local partnership if issues of privacy and transparency were not properly addressed. Instead of merely creating a privacy functionality within the dashboard (e.g. a "share / don't share" toggle), this situation prompted a research project that could reveal deeper issues of privacy, transparency and trust across our partner schools. This logic motivated our choice for an iterative and participatory design process that could be documented and later presented to the LA community. Our choice for design-based research $[6,14,37]$ allowed us to go beyond simply developing a set of front-end features and, instead, theorize about how tools and their associated structures and discourses could meet or even re-frame ethical aspects of our partnerships.

\subsection{Analytical Frameworks}

To elicit and reflect upon issues of privacy, transparency and trust, we adopted two main research frameworks:

3.2.1 Research th rough Design. We utilized Zimmerman et al.'s [45] research-through-design model to frame our study. Zimmerman and colleagues outline four aspects that need to be present when researchers seek to make a theoretical contribution through design work: process, invention, relevance, and extensibility. Process refers to thick descriptions of the design process and method of analyses. Invention describes how the proposed design incorporates insights from theory and practice to address a problem. In our work, we documented the tensions that arose with each prototype, built on emergent literature around privacy, and proposed design rationales and core design ideas to address those tensions. Each prototype addresses specific design issues of previous versions. Regarding Relevance, Zimmerman and colleagues suggest that design researchers provide "traces" for the scholarly community by documenting the motivations and context that justify their work. This principle is precisely what we sought in each of the four design narratives presented below, which illuminate the perspectives of multiple stakeholders (instructional coaches, district leaders, researchers, designers) to describe how the prototypes connected to specific contexts. Finally, Extensibility describes how the outcomes of a design study might expand to similar sets of problems. In the Discussion section, we present conjectures of how to promote trust, transparency and privacy in data sharing routines in schools.

3.2.2 Conjecture Maps. Conjecture mapping [31] as utilized in the learning sciences allows researchers to systematically hypothesize the flow between material aspects of a design ("embodiments"), key mediating processes, through which the design presumably supports teaching and learning, and intended learning outcomes. In his framework, Sandoval [31] describes two types of conjectures: design conjectures are the assumed chains that explain how embodiments might generate mediating processes, whereas theoretical conjectures explain how such processes might generate outcomes. In other words, conjecture mapping represents how designed elements are theorized to work together to produce the desired outcomes.

We chose to marry conjecture mapping with a research-throughdesign framework, because conjecture maps offer a concise way to communicate process, invention, relevance, and extensibility. Conjecture mapping is not only suitable for narrating design in hindsight but, above all, informing future design research efforts $[31,40]$. This view is particularly beneficial for iterative and participatory design work such as the present study, where researchers can test design and theoretical conjectures successively. For this specific purpose, Sandoval [31] recommends developing "a sequence of conjecture maps (...) in which particular design or theoretical relationships are deleted, modified, or added" (p. 33). In our study, we employed conjecture mapping as both a research road map - where specific facets of the design and participant interactions where mapped vis-a-vis their desired outcomes - and as a data analysis tool, allowing for retrospective reflections about the design sequence. While Figure 3 shows the map representing our team's initial conjectures, Figure 8 is used to guide our discussion and lay out future research and design efforts.

\subsection{Data Sources}

To create a thick description of our design process [14], this study uses a wide corpus of data (Table 1). First, we catalogued design notes from weekly meetings with our design team - comprising researchers, interaction designers and developers - to document the evolution of our conjectures for each prototype. Some of these notes were taken directly on Figma, a User Interface Design software, and pointed to assumptions, reflections and potential design changes. One example of a design note is "The key tension is how complicated [it is for] educators make choices. Sharing individually is more complicated but gives more control" (Design notes, April 2020). Second, taking advantage of our research network's annual meetings, we conducted two co-design sessions with researchers and partner educators, including teachers, coaches and district specialists, to identify problems of practice that could inform the design of the dashboard. To elicit and capture issues of privacy and trust, we used a number of design techniques in these sessions, including stickynoting, recording of verbal and written feedback to low-fidelity 


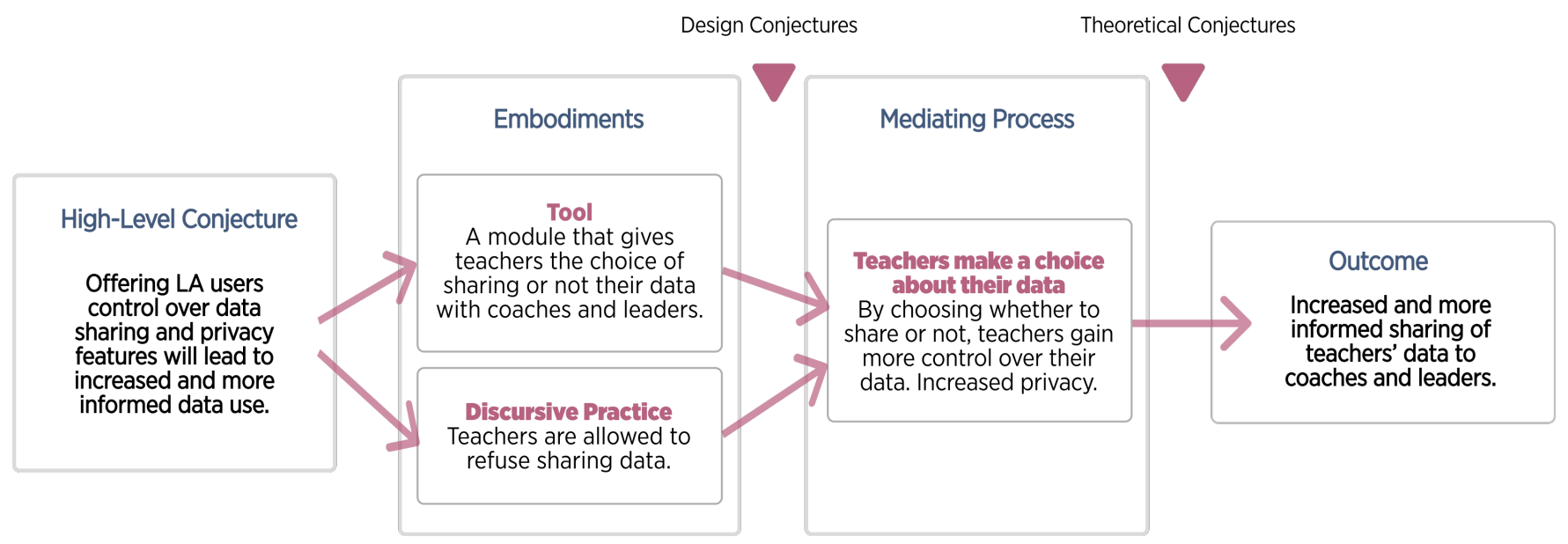

Figure 3: Map of initial conjectures, made prior to the design process.

prototypes, and mapping of user journeys. These artifacts captured the context and work routine in our partner schools and helped to ground our design ideas. The audio-taped discussion from these sessions deeply informed the design rationales of our prototypes.

Based on our design notes and larger co-design sessions with partner districts, we developed an initial set of low-fidelity prototypes, and mapped our conjectures for how specific features theoretically link to desired outcomes (see Figure 3 for example). Finally, we conducted three semi-structured group interviews with two district leaders and five instructional coaches from two partner school districts. Participants were presented with prototypes 1-3, and asked to think aloud about how different prototype versions might play out in their school contexts, and what their daily workflow could look like with different tools. The interviews revealed insights into educators' perceptions of privacy, transparency, and trust dynamics in their respective school environments. We used the interview protocol, audio-recordings of the interviews, and researcher memos, to explore the affordances and constraints of each prototype and, finally, developed Prototype 4 as a final embodiment of our evolving learning.

\subsection{Analytical Procedures}

To analyze our wide corpus of data, our research team conducted four steps. First, we organized all data sources in one single corpus, differentiating events such as co-design workshops, interviews, research team meeting and design notes. Then, we conducted two consecutive passes of coding of each excerpt or meeting note [25]. In the first pass, we generated low inference codes such as "conversation", "hierarchy", "negotiation" and "trust". Four coders participated in this stage and agreement was established by internal meetings of the team. In the second pass, we grouped codes into inferential units that connected the ideas present in the excerpt. Examples include "conversation versus evaluation" and "leveled privacy." Third, we wrote memos for each data event (i.e. interview, meeting, etc.), which summarized the main ideas revealed by codes. Finally, we organized all memos and notes into a map of tensions, design solutions and push-backs, and retrospectively rebuilt the design narratives this paper presents. In this paper, we present design narratives of the different iterative prototypes our partnership team developed, and a series of conjecture maps that document the implicit theories of design, embodiment, interaction, and desired outcomes that the prototypes reveal. By grounding the design work - and analysis of this process - in the actual realities of our practice partners, we provide a methodology to qualitatively theorize about the issues of privacy, transparency, trust that are present in K-12 school settings.

\section{DESIGN NARRATIVES}

In this section, we present the co-development of four prototypes as thick design narratives [14]. In essence, we seek to "make explicit some of the implicit knowledge the designer or designer-researcher used to understand and implement the intervention" (p. 454). We start each narrative by describing the theoretical considerations and implicit assumptions that motivate our design, followed by the insights gained after testing each prototype with our partners.

We began designing the first prototype with a core tension that was common in our partner schools. On the one hand, teachers in our partner schools were expected to share all of their data (e.g., notes, surveys, and classroom artifacts such as lesson plans) with their instructional coaches, illustrated by a coach when she stated: "If a teacher agrees to be coached, there is an expectation that they will share their data with their coach" (Coach, West District; Interview May 2020). On the other hand, some of our partner district leaders voiced the concern that teachers would experience an oppressive lack of agency if they were "forced", or assumed to have to share all of their data, with no choice in the matter. Thus, we began the iterative design process with an initial conjecture to develop a tool to give teachers a choice in sharing their data or not with other stakeholders in their school or district.

\subsection{Prototype 1: "All or Nothing"}

In Prototype 1 (Figure 4, teachers were presented with the choice to share their data and artifacts with coaches or district math leaders; 
or to keep their data private. The main affordance of Prototype 1 was that it presented a simplified choice of sharing all versus sharing none of a teacher's data. This initial prototype drew directly from the initial interviews and needs voiced by our partners.

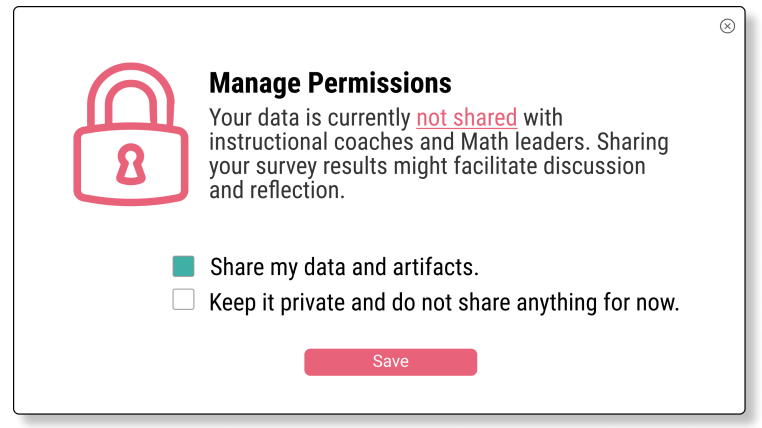

Figure 4: Prototype 1: “All or Nothing”

However, our co-design sessions with partner researchers and educators revealed new tensions. Researchers, coaches, and instructional leaders expressed concerns that the teachers may not share data at all if given such choice. For example, District Leader C, from West District (Interview, May 2020), voiced that "it could be a lost opportunity if half your teachers opted out of sharing that data". Conversely, one of the co-design participants suggested that the prototype gave teachers no control, as teachers might feel they had no choice but to agree to share data simply due to compliance and accountability pressure (Design Notes, June 2020). These tensions have been widely documented in the literature on teacher data sharing practices. For example, Little [19] notes that there exists a continuum of professional relationships that influences the extent to which teachers are willing to share data. Meanwhile, LA scholars have highlighted that users should be agents of data use, and not merely resign their data to those with more authority [35, 38].

Observations of these frictions led our team to a key insight: Our design should give teachers more choice in how they share, but mitigate the situation of not sharing any data (which would derail any data-driven initiative at the outset), and also address workplace situations where teachers may be given undue, toxic pressure to share their data. The interviews and design meetings provided us with a multitude of ideas related to why teachers would want to share some data with certain school district personnel but not with others. One of our partner coaches (West District) mentioned that a teacher may be comfortable sharing data with an instructional coach, but not necessarily the school principal. This information suggests that data sharing practices may directly depend on school roles and group norms. For example, in a given school, the main role of an instructional coach could be viewed as a supportive, non-evaluative role, to help teachers to improve their practice, whereas, a principal may have direct influence on educators' work evaluations, and thus be seen as the instruments of district or even state-wide accountability practices.

Our partner coaches also pointed to concerns around sharing specific types of data. For example, teachers would be less willing to share unpolished, personal notes that they made to themselves about specific classroom practices. This motivated our team to think of ways (in Prototype 2) to keep teachers' notes private by design, despite their usefulness for coaches when making sense of pedagogical moves. These insights also illustrate that the practice of data sharing within a K-12 school may be nuanced and complex, and not simply conforming to hierarchical norms and expectations. We began to experiment with Prototype 2, where we provided teachers with more fine-grained control over what types of data to share and with whom they could provide access to their information.

\subsection{Prototype 2: "What and with Whom"}

Following the insights gained through Prototype 1 - that hierarchical relations might impact the nature of data sharing - we developed and tested a second prototype (Figure 5). In Prototype 2, teachers can choose to share specific types of data for different educator roles. Prototype 2 was designed based on the idea that depending on how roles were understood in a given school context, teachers had agency to share specific types of information. For example, our partner educators voiced that instructional coaches should receive detailed teacher data, as they are tasked to help teachers with instructional decisions. Conversely, district-level staff should receive aggregate data (blinding teacher names and classroom identifiers) to safeguard teachers.



Figure 5: Prototype 2: "What and Whom".

One major tension became clear when we tested Prototype 2 with our partners: sharing options were still not granular enough to provide teachers with the necessary space to share only the data they deemed necessary. For example, when teachers selected options such as "share my survey results", all data events, from all classes and school years, were shared with coaches or district personnel. Our partner educators also pointed out the fact that sharing data based on school and district roles was a step further toward teachers' agency but still fell short of providing the level of trust and control that our local research practice partnerships envisioned. Consider how District Leader L described how she expected conversations to be at the core of coach-teacher relations when working with data:

If I were a school coach, I would probably have a conversation with the teacher on what they want to work on and grow for the year" (L., West District, Co-Design session, May 2020) 
Leader L illuminates the need to move beyond sharing based on hierarchical roles, and amplify conversations between stakeholders to build understanding around data sharing tasks. This line of thought echoed a preceding recommendation made by one researcher who reacted to initial prototypes of the dashboard by stating the following:

"[We need to] switch from a 'technocratic', prescriptive way to tell teachers and coaches how to [work] to having more conversations, and [provide] trust/agency for users" (Researcher, Design Notes)."

Reflections such as the one above, made consistently by both researchers and partner educators throughout the design process, motivated the design development of the "data initiative" concept, materialized through Prototype 3.

\subsection{Prototype 3: "Data Initiatives"}

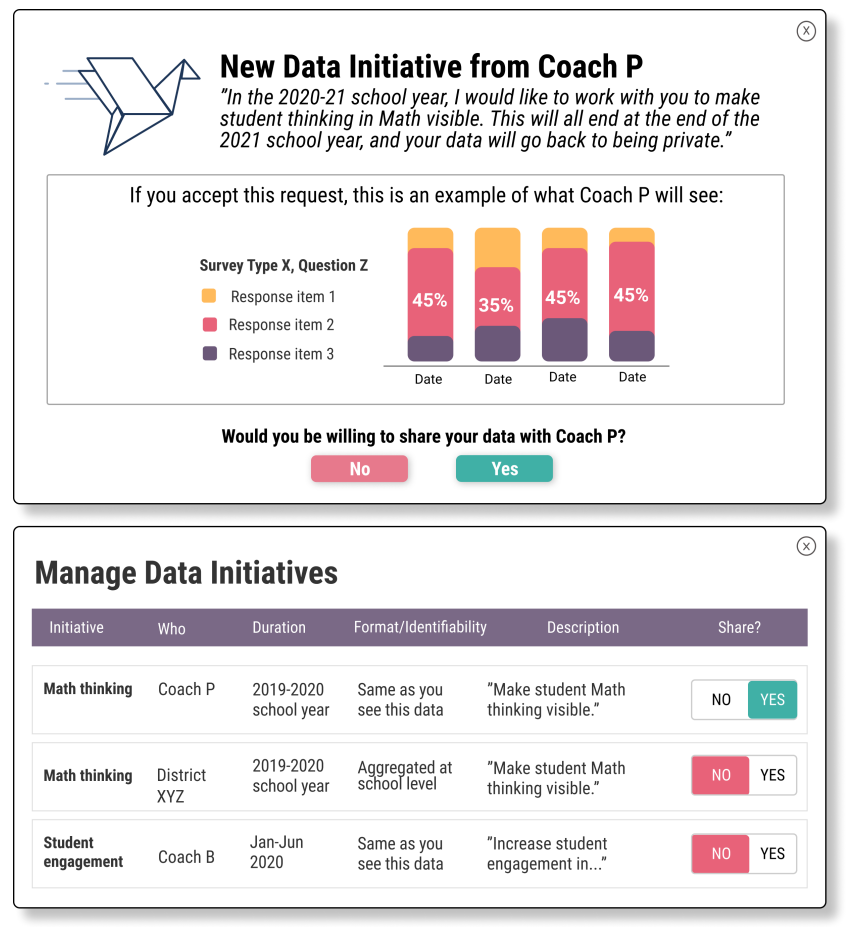

Figure 6: Prototype 3: a teacher receives a data initiative request (top) and manages multiple requests (bottom).

The underlying assumption for Prototype 3 was that transparency about instructional improvement goals may drive data sharing practices between educators. In this prototype, stakeholders "requested" data from each other, and in the process of this request must "describe to stakeholders the processes by which the data are handled" ([27] p. 446). In the words of Coach D (Interview, May 2020), this design mapped onto their existing routine, "for coaches to share [with teachers] their problems of practice, questions, wondering, goals, celebrations." With Prototype 3 (Figure 6), educators' roles within a school or district were still central to the logic of who would share data with whom. What also stayed constant was the granularity of what can be shared: coaches can request classroom-level data from teachers, but district leaders can only request aggregated, non-identifiable student data.

However, three major changes characterize Prototype 3. First, we redesigned the task associated with data sharing. While the previous versions had privacy and data sharing as a setting, materialized by check-boxes and always initiated by the teacher, Prototype 3 introduced the mechanic of data requests initiated by the coach and later authorized by the teacher. Second, we added to this mechanic several discursive components, such as asking coaches and district leaders to clearly justify the reason for the request. For example, if a coach and teacher were working on a specific math practice that involved student surveys, class artifacts, and teacher notes, the coach had to provide a reason (e.g., a goal to improve a specific practice) for why they were asking teachers to share access to those various types of data. Third, we included a preview of how teacher's data would be displayed to the requester, as seen on Figure 6. Together, these three design changes resulted in what we later called a data initiative, or a request for teachers' data bounded by a reason $[9,29,35]$, a duration (after which the requester cannot see the data) $[9,27]$ and a preview of how data would be seen.

With Prototype 3, however, tensions around school hierarchy remained. The new task design assumed a fixed participant structure [31] where teachers were data providers and coaches or district leaders were analysts of classroom data. Teachers did not have any way to initiate data sharing themselves, but only to accept or refuse requests. A key insight that emerged in testing Prototype 3 was that trust and privacy cannot be expected from an unidirectional process that emphasizes hierarchical, fixed roles. Thus, we explored multi-directional data sharing practices in Prototype 4.

\subsection{Prototype 4: "Multi-directional Sharing"}

We started the design of Prototype 4 (Figure 7) based on the premise that conversations and negotiations should foreground data sharing for professional improvement. In this prototype, features from iterations 2 and 3 were combined: teachers could still select what to share, and preview how their data would be displayed. We also introduced elements that spoke to the hierarchy between educator roles (Figure 7). First, the prototype allows for multi-directional data sharing. The interface includes two tabs for "Shared with me" and "My data" - the former being data that teachers have requested from others, and the latter being data that teachers are sharing. This interface decision means that teachers could request data from other teachers in the same inquiry cycle, where coaches and teachers collaboratively work on an instructional goal, or choose to share data on their own, without coaches or districts' initiating the process. Second, we included built-in design choices to facilitate ongoing conversations and negotiations among educators about the need and potential uses of data. For example, when a coach, a teacher, or a district specialist requested or shared data, she would be prompted to provide the overarching goal for the request. Similarly, when an educator declined a data sharing request, the interface would present her with the option to provide an explanation for this decision. These designs can prompt users to reflect on their decisions and to transparently communicate their perceptions and intentions for data use to other stakeholders. Additionally, we 


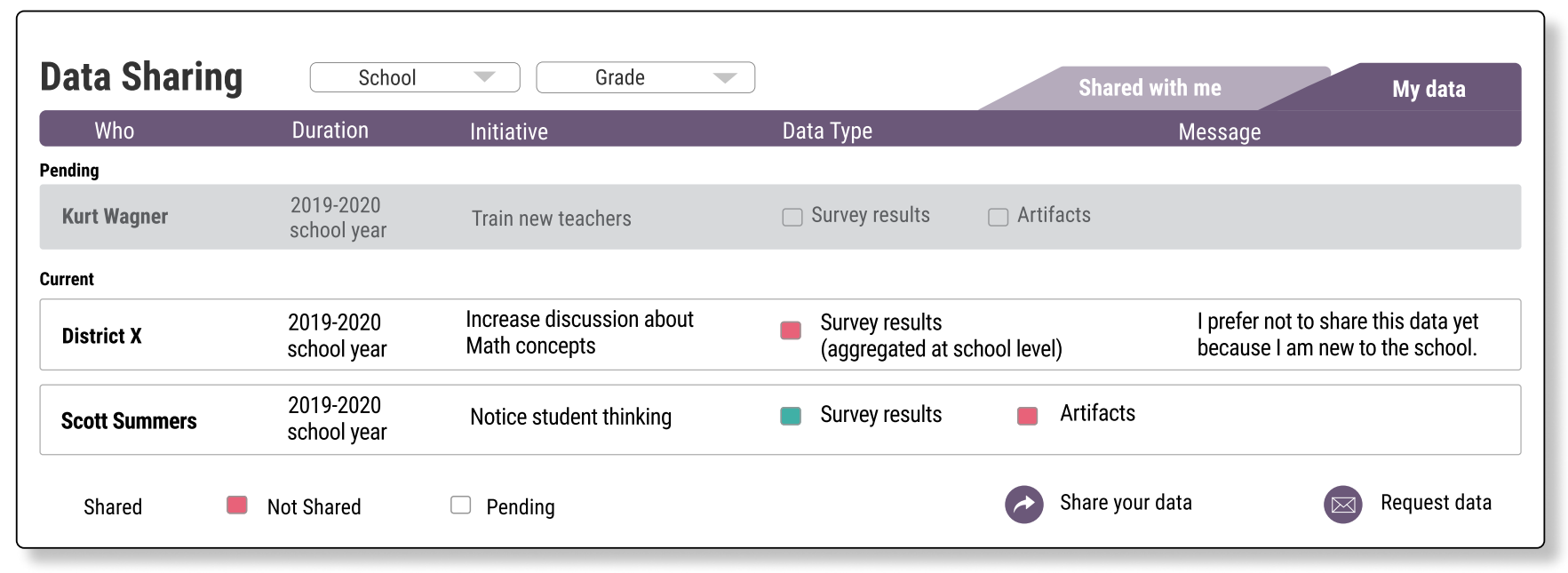

Figure 7: Prototype 4: users can request from others and share their own data.

removed the role of the requester (e.g., coach, district leader), thus leaving only their names under "who" teachers were sharing data with. This personal-touch intervention suggests that there should be an offline relationship between stakeholders prior to any data sharing procedures in the platform.

These design decisions were noticed by the broader research team embedded in district partnerships who raised questions such as "After a teacher declines a request, can s/he go back and decide to share?" (Design Meeting, July 2020). This quote suggests an awareness of the the data use experience, where trust is established through ongoing interpersonal interactions. Another researcher noted how such a process would lead to meaningful conversations around data sharing. In his words, "[with a] data request, there is room for conversation. Are you sure you want to request it? Is it clear what you are requesting?" (Design Meeting, July 2020).

In the next section, we reflect on our successive iterations, map the conjectures surrounding Prototype 4, and outline how theoretical and material facets of our design are expected to support data sharing for improvement practices.

\section{DISCUSSION}

LA and HCI scholars have made significant efforts in developing frameworks and codes of ethical practice. In our work, we aimed at putting into practice such ethics frameworks by co-designing privacy tools that promote data sharing with privacy, transparency and trust. By employing an iterative design method, we discovered how intentionally designing tasks, discourses and participant structures - and not simply tools or features in a system - revealed key ways to elicit transparency and trust for our partners' [43] within a school organizations. By doing so, this study attempts to move beyond checklist approaches [42] to intentionally design, test and refine LA tools that are responsive to the local and contextually-bound views $\mathrm{K}-12$ educators have of ethics $[11,36]$.

In the next subsection, we analyze how the design process allows us to rethink our conjectures about how K-12 educators approach trust, privacy and transparency in collective data sharing practices.

\subsection{Refining Conjectures around Data Sharing}

Following Sandoval's [31] notion of conjecture mapping - and inspired by Wilkerson's [40] iterative use of such tools to explain incremental design decisions - our team refined the initial conjectures and assumptions we brought to this design process to build an updated understanding of the realities of our partners. Figure 8 presents our revised conjecture map, detailing how the findings from the design of iterations 1 to 4 informed our final understanding of how technological tools could support improved data privacy, transparency, and trust for our K-12 partners.

5.1.1 Outcome. The tensions around trust building, which arose from the earlier prototypes, guided us to revise the overall Outcome of the design. Attending to users' values prompted us to include mechanisms for transparency that contributed to trustbuilding processes between users. Understanding that transparency must go beyond compliance and informed consent [27], we theorize a second overarching outcome: a refined understanding about data-driven pedagogical improvement. In other words, we conjecture how meaningful conversations and deliberate engagement towards data sharing would serve as learning tools about how information is used to improve instruction.

5.1.2 Mediating Processes. The insights gained from our partners revealed a tension between school hierarchy versus interpersonal trust. Why should only coaches and district leaders launch data requests? Would this lead to monitoring and surveillance? Why should teachers be put in a gatekeeper position, merely accepting or refusing to share their own data? Understanding that trust among peers and between individuals and institutions might be conducive to actual data disclosure [26], we revised our understanding of which mediating processes should be achieved to ultimately enable the revised outcomes.

Our new theoretical conjectures aimed to create a multi-party, multi-directional process of Deliberate Engagement to build collective understanding about data use. We define Deliberative Engagement as the process of conscientious, informed and voluntary 


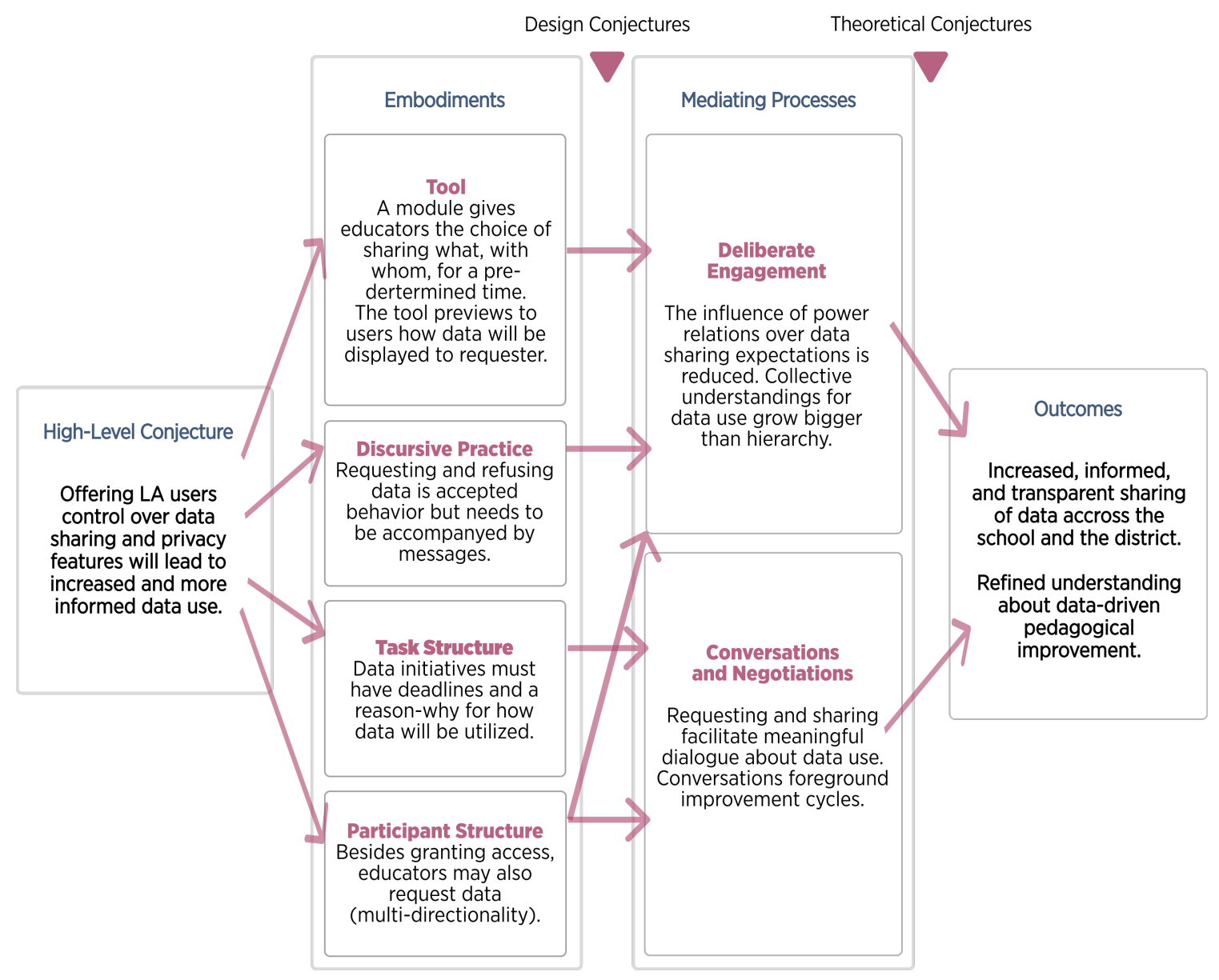

Figure 8: Map of conjectures produced at the end of the design process.

participation in a data sharing routine, where teachers are also able to initiate requests for their own improvement purposes. We note that deliberate engagement does not change or challenge hardwired hierarchies, but attenuate the negative effects they can have over sharing expectations, privacy and trust. We also conjectured how Conversations and Negotiations around sharing and privacy and potential uses of data should necessarily foreground school and district based improvement cycles. Through this process, our team theorized not only an increased but, most importantly, a more informed data sharing behavior among stakeholders.

5.1.3 Embodiments. To achieve the above processes, we updated which Embodiments would need to be redesigned or integrated. Feedback from educators and researchers provided insights into how trust is not a fixed construct based on hardwired hierarchies or roles, but is constantly negotiated between stakeholders. Based on this view, our prototypes journeyed from a simple system setting - where teachers make decontextualized choices about data sharing - to a tool that requires dialogue within and outside of the platform. Moreover, we learned that this central change could not be achieved by the tool alone (i.e. the privacy module on a larger dashboard), but needed supports not previously designed on
Conjecture Map 1 (Figure 3). For this reason, we understood the need to redesign the data sharing 1) task structure to represent our data initiatives, 2) the discursive practices around sharing, where reasons for sharing or requesting became canonical practice, and 3) the participant structure, to reflect the role played by multi-directional sharing initiatives. Making explicit the types of data being shared and the purposes they serve helps make the tool and sharing practices more transparent. The ongoing nature of the discursive practice and task structure in the revised map (Figure 8), where educators can go back and provide reasoning for requesting and refusing data, reflects the iterative nature of data sharing in schools.

\subsection{Design as Research}

We draw from a research-through-design methodology [45] to discuss how sequential design work, grounded in the context of K-12 educators, might contribute to the practice and research of LA.

5.2.1 Process. First, to make explicit our research process, we developed thick design narratives [14] that shed light on the implicit knowledge of the work context, design principles, and dialectics present in the school workplace of our partners. We note how 
conjecture maps might be employed by the LA community to track how ethical codes of practice might translate into designs, and which processes are expected from such embodiments. We posit that, without such a process, issues of ethics might be limited to the plane of principles or checklists without being put to practice through design in real learning contexts.

5.2.2 Relevance. Considering that all human-made tools carry the assumptions and choices of their creators [41], opening the black box of decisions, motivations and tensions behind the design of LA platforms is of utmost importance. The recognition that issues of "trust raises ethical questions for the design and implementation of LA" ([13], p. 51) illuminates the need and relevance of an informed and transparent design process in LA. In addition, by grounding our design work in partnership with educators in three districts, we ensured that our process is also directly and practically relevant (or tuned) to specific practitioners and use.

5.2.3 Invention. Our team incorporated insights from both theory and practice throughout the process that led us to Prototype 4. From a theoretical perspective, we heed the call for increased privacy and trust in computer mediated processes in the workplace $[7,8,24]$. Understanding the school both as a workplace - prone to micropolitical forces and power struggles $[18,19]$ - and as part of a larger system (i.e. the district), was instrumental for our design process. We also understood the importance of making an often peripheral topic - privacy - central not only for increased data sharing but also a value lever [43], conducive to deeper understandings of data-driven pedagogical improvement efforts [17]. In this sense, we were able to shed light on how conversations around data privacy can be theorized as potential contributors to larger data-literacy efforts being conducted in school districts across the United States [22].

5.2.4 Extensibility. Finally, we view our later prototypes and their associated tasks, structures and processes, as highly extensible to the larger LA community. Proximity with other users, group norms and knowledge about data sharing purposes have been mapped by Pardo and Siemens [27] as central to fostering trust in learning institutions. In facing privacy, trust and data-sharing challenges in schools or universities, LA researchers and practitioners might need to refrain from the widely present discourse of algorithmic neutrality [34] to consider how ethics and privacy decisions are highly transient and contextual [11]. Understanding that issues of agency and control over data are not exclusive to K-12 settings, we illuminate how dialogue and deliberate engagement might attenuate distrust and reduce tensions between stakeholders of a learning institution.

Moreover, our conjecture maps serve as open-ended artifacts extensible, testable, and usable to our research community. We invite scholars to explore analogous sets of conjectures in other research contexts, and recreate said maps, as a means to test, reflect upon and put to practice the frameworks and codes of practice produced by our community.

\section{STUDY LIMITATIONS}

We note two limitations that contextualize and bound the analyses presented here. First, our conclusions are grounded in the context and problems of practice of the districts where the study was conducted. Second, our design-based approach was intended to reveal aspects of privacy and trust through multiple iterations of a prototype, and not to evaluate the adoption of the resulting tool by partner districts, which will be the focus of a future case study.

\section{CONCLUSION AND NEXT STEPS}

Our work developing a data sharing and privacy prototype began with a set of implicit design assumptions culled from the intersection of extant LA and HCI research, as well as our own research team's experiences. Building from these initial conjectures, our co-design partners from our focal schools provided critical perspectives on issues surrounding privacy, transparency and trust within the K-12 educational context. Without their contributions, important and nuanced insights would have been missed, potentially jeopardizing the use and longevity of the larger dashboard for which the privacy module is being created. Our use of conjecture maps - and our ongoing revision to these maps - anchored our design choices and provided common ground for our research team to explore and play with potential changes to our prototypes. Most importantly, what became clear from this project is that the interactive, co-design nature attuned all stakeholders (designerresearchers, teachers, coaches, district leaders) to issues of privacy, transparency and trust, suggesting the importance of attending to the values and social norms of the communities for which we are designing.

\section{ACKNOWLEDGMENTS}

This material is based upon work supported by the National Science Foundation under grants No. 1719744, 1620851, 1621238 and 1620863. Any opinions, findings, and conclusions or recommendations expressed in this material are those of the author(s) and do not necessarily reflect the views of the NSF.

\section{REFERENCES}

[1] June Ahn, Lauren K. Bivona, and Jeffrey Discala. 2011. Social media access in K-12 schools: Intractable policy controversies in an evolving world. Proceedings of the ASIST Annual Meeting 48 (2011). https://doi.org/10.1002/meet.2011.14504801044

[2] June Ahn, Fabio Campos, Maria Hays, and Daniela DiGiacomo. 2019. Designing in Context: Reaching Beyond Usability in Learning Analytics Dashboard Design. fournal of Learning Analytics 6, 2 (8 2019), 70-85. https://doi.org/10.18608/jla. 2019.62 .5

[3] Suzanne Blanc, Jolley Bruce Christman, Roseann Liu, Cecily Mitchell, Eva Travers, and Katrina E. Bulkley. 2010. Learning to learn from data: Benchmarks and instructional communities. Peabody fournal of Education 85, 2 (2010), 205-225. https://doi.org/10.1080/01619561003685379

[4] Simon J. Buckingham Shum and Rosemary Luckin. 2019. Learning analytics and AI: Politics, pedagogy and practices. British fournal of Educational Technology 50, 6 (2019), 2785-2793.

[5] Bodong Chen and Haiyi Zhu. 2019. Towards Value-Sensitive Learning Analytics Design. In Proceedings of the 9th International Conference on Learning Analytics \& Knowledge - LAK19. 343-352. http://arxiv.org/abs/1812.08335

[6] Paul Cobb, Jere Confrey, Andrea Disessa, Richard Lehrer, and Leona Schauble. 2003. Design Experiments in Educational Research. Educational Researcher 32, 1 (2003), 9-13. https://doi.org/10.3102/0013189X032001009

[7] Christina J. Colclough. 2018. Not Just a Facebook Problem: Ethical data collection must be employed at work. https://www.oecd-forum.org/posts/32785-not-justa-facebook-problem-ethical-data-collection-must-be-employed-at-work

[8] Regina Connolly and Cliona McParland. 2012. Dataveillance: Employee monitoring \& information privacy concerns in the workplace. fournal of Information Technology Research (FITR) 5, 2 (2012), 31-45.

[9] Hendrik Drachsler and Wolfgang Greller. 2016. Privacy and analytics - it's a DELICATE issue a checklist for trusted learning analytics. ACM International 
Conference Proceeding Series 25-29-Apri (2016), 89-98. https://doi.org/10.1145/ 2883851.2883893

[10] Julie A. Farrell, Caitlin C.; Marsh. 2016. Contributing conditions: A qualitative comparative analysis of teachers' instructional responses to data. Teaching and Teacher Education 60 (11 2016), 398-412.

[11] Rebecca Ferguson, Tore Hoel, Maren Scheffel, and Hendrik Drachsler. 2016. Guest editorial: Ethics and privacy in learning analytics. Fournal of Learning Analytics 3, 1 (2016), 5-15. https://doi.org/10.18608/jla.2016.31.2

[12] Dragan Gašević, Shane Dawson, and Jelena Jovanović. 2016. Ethics and Privacy as Enablers of Learning Analytics. Journal of Learning Analytics 3 (2016), 1-4.

[13] Dai Griffiths. [n.d.]. The Ethical Issues of Learning Analytics. In Radical Solutions and Open Science. Lecture Notes in Educational Technology., D. Burgos (Ed.) Springer Singapore, Chapter 3, 39-55. https://doi.org/10.1007/978-981-15-4276-3

[14] Christopher Hoadley. 2002. Creating context: Design-based research in creating and understanding CSCL. Engineering Education (2002), 453-462.

[15] Ilana Seidel Horn, Britnie Delinger Kane, and Jonee Wilson. 2015. Making Sense of Student Performance Data. American Educational Research fournal 52, 2 (2015), 208-242.

[16] Kara Jackson, Erin Henrick, Paul Cobb, Nicholas Kochmanski, and Hannah Nieman. 2016. Practical measures to improve the quality of small-group and wholeclass discussion. White Paper (2016), 1-8. http://www.education.uw.edu/pmr/ files/2016/09/White-Paper.pdf\%0AIntroduction

[17] Priya C. Kumar, Marshini Chetty, Tamara L. Clegg, and Jessica Vitak. 2019. Privacy and security considerations for digital technology use in elementary schools. Conference on Human Factors in Computing Systems - Proceedings 1 (2019), 1-13. https://doi.org/10.1145/3290605.3300537

[18] Judith Warren Little. 1990. The Persistence of Privacy: Autonomy and Initiative in Teachers? Professional Relations Making Sense of Science, in collaboration with WestEd View project. Teachers College Record 91, January 1990 (1990), 509-536.

[19] Judith Warren Little. 2012. Understanding data use practice among teachers: The contribution of micro-process studies. American fournal of Education 118, 2 (2012), 143-166. https://doi.org/10.1086/663271

[20] Ellen B. Mandinach. 2012. A Perfect Time for Data Use: Using Data-Driven Decision Making to Inform Practice. Educational Psychologist 47, 2 (2012), 71-85. https://doi.org/10.1080/00461520.2012.667064

[21] Ellen B. Mandinach and Edith Gummer. 2015. Data-driven decision making: Components of the enculturation of data use in education. Teachers College Record 117, 4 (2015), 1-8.

[22] Ellen B. Mandinach and Jo Beth Jimerson. 2016. Teachers learning how to use data: A synthesis of the issues and what is known. Teaching and Teacher Education 60 (2016), 452-457. https://doi.org/10.1016/j.tate.2016.07.009

[23] Julie A. Marsh, Jennifer Sloan McCombs, and Francisco Martorell. 2010. How Instructional Coaches Support Data-Driven Decision Making. Educational Policy 24, 6 (2010), 872-907. https://doi.org/10.1177/0895904809341467

[24] Cliona McParland and Regina Connolly. 2020. Dataveillance in the Workplace: Managing the Impact of Innovation. Business Systems Research 11, 1 (2020), 106-124. https://doi.org/10.2478/bsrj-2020-0008

[25] Matthew B Miles and A. Michael Huberman. 1994. Qualitative data analysis: an expanded sourcebook. SAGE, Thousand Oaks, CA.

[26] Patricia A. Norberg, Daniel R. Horne, and David A. Horne. 2007. The privacy paradox: Personal information disclosure intentions versus behaviors. , 100126 pages. https://doi.org/10.1111/j.1745-6606.2006.00070.x

[27] Abelardo Pardo and George Siemens. 2014. Ethical and privacy principles for learning analytics. British fournal of Educational Technology 45, 3 (2014), 438-450. https://doi.org/10.1111/bjet.12152

[28] Rita Prestigiacomo, Roger Hadgra, Jane Hunter, Lori Locker, Simon Knight, Elise Van Den Hoven, and Roberto Martinez-maldonado. 2020. Learning-centred Translucence : An Approach to Understand How Teachers Talk About Classroom Data. In Proceedings of the Tenth International Conference on Learning Analytics \& Knowledge - LAK20. 100-105.

[29] Paul Prinsloo and Sharon Slade. 2017. Handbook of Learning Analytics. Handbook of Learning Analytics (2017).

[30] Peter Reimann. 2016. Connecting learning analytics with learning research: the role of design-based research. Learning: Research and Practice 2, 2 (2016), 130-142. https://doi.org/10.1080/23735082.2016.1210198

[31] William Sandoval. 2014. Conjecture Mapping: An Approach to Systematic Educational Design Research. Fournal of the Learning Sciences 23, 1 (2014), 18-36.

[32] Kim Schildkamp. 2019. Data-based decision-making for school improvement: Research insights and gaps. Educational Research 61, 3 (2019), 257-273.

[33] Niall Sclater. 2016. Developing a Code of Practice for Learning Analytics. Fournal of Learning Analytics 3 (2016), 16-42.

[34] Neil Selwyn. 2019. What's the problem with learning analytics? fournal of Learning Analytics 6, 3 (2019), 11-19. https://doi.org/10.18608/jla.2019.63.3

[35] Sharon Slade and Paul Prinsloo. 2013. Learning Analytics: Ethical Issues and Dilemmas. American Behavioral Scientist 57, 10 (2013), 1510-1529.

[36] Sharon Slade, Paul Prinsloo, and Mohammad Khalil. 2019. Learning analytics at the intersections of student trust, disclosure and benefit. ACM International Conference Proceeding Series (2019), 235-244.

[37] The Design-Based Research Collective. 2003. Design-Based Research: An Emerging Paradigm for Educational Inquiry. Educational Researcher 32, 1 (2003), 5-8. https://doi.org/10.3102/0013189X032001005

[38] Yi-Shan Tsai, Alexander Whitelock-Wainwright, and Dragan Gašević. 2020. The privacy paradox and its implications for learning analytics. In Proceedings of the Tenth International Conference on Learning Analytics \& Knowledge, LAK20. 230-239. https://doi.org/10.1145/3375462.3375536

[39] Josine Verhagen, Lucie Dalibert, Federica Lucivero, Tjerk Timan, and Larissa Co. 2018. Designing values in an adaptive learning platform. International Learning Analytics \& Knowledge Conference (2018). https://halshs.archivesouvertes.fr/halshs- 01650224

[40] Michelle Hoda Wilkerson. 2018. Teachers, Students, and After-School Professionals as Designers of Digital Tools for Learning. Participatory Design for Learning (2018), 125-138. https://doi.org/10.4324/9781315630830-13

[41] Langdon Winner. 1980. Do Artifacts Have Politics? Daedalus 109, 1 (1980), 121-136.

[42] Richmond Y Wong and Deirdre K Mulligan. 2019. Bringing Design to the Privacy Table: Broadening "Design" in "Privacy by Design" Through the Lens of HCI. In CHI Conference on Human Factors in Computing Systems Proceedings (CHI 2019). 17. https://doi.org/10.1145/3290605.3300492

[43] Richmond Y. Wong, Deirdre K. Mulligan, Ellen Van Wyk, James Pierce, and John Chuang. 2017. Eliciting values reflections by engaging privacy futures using design workbooks. Proceedings of the ACM on Human-Computer Interaction 1, CSCW (2017). https://doi.org/10.1145/3134746

[44] David Yeager, Anthony Bryk, Jane Muhich, and Lawrence Morales. 2013. Practical Measurement.

[45] John Zimmerman, Jodi Forlizzi, and Shelley Evenson. 2007. Research through design as a method for interaction design research in HCI. Conference on Human Factors in Computing Systems - Proceedings (2007), 493-502. https://doi.org/10. $1145 / 1240624.1240704$ 